\title{
FTIR Analysis for Quantification of Fatty Acid Methyl Esters in Biodiesel Produced by Microwave-Assisted Transesterification
}

\author{
Sabrina N. Rabelo, Vany P. Ferraz, Leandro S. Oliveira, and Adriana S. Franca
}

\begin{abstract}
Biodiesel was produced by microwave-assisted transesterification of soybean oil with methanol as esterifying agent and sodium methoxide as catalyst. Fourier transform infrared spectroscopy was employed as a fast and reliable analytical technique for the quantification of fatty acid methyl ester content in the produced biodiesel. The quantification was done with the use of a partial least squares model developed based on the infrared spectra obtained. It was shown that microwave irradiation is capable of reducing the reaction time when compared to conventional mechanically stirred reactors used for biodiesel production. In addition, quantification of fatty acid methyl ester content in biodiesel by Fourier transform infrared spectroscopy coupled to multivariate statistics was demonstrated feasible.
\end{abstract}

Index Terms-Biodiesel, microwave, FTIR.

\section{INTRODUCTION}

The transesterification of vegetable oils in batch processes is the most commonly used technology for biodiesel production, in which a short chain alcohol reacts with the oil in a stirred tank to produce the alkyl esters of fatty acids (biodiesel), with a basic homogeneous catalyst being used to accelerate the reaction [1]. One of the major advances in technology for the biodiesel production of recent times is the employment of microwave-irradiated reactors for the transesterification of oils, in which the reaction times are significantly reduced when compared to the conventional processes [2]. The effects of microwave irradiation on the transesterification reactions were studied theoretically and experimentally by Asakuma et al. [3], where triolein was used as representative of the triglyceride class in oil. It was concluded that it is not only thermal effects that improve the reaction rates but also modifications that occur in the stereochemistry of triglycerides molecules under irradiation. It was theoretically demonstrated the a planar triolein was formed under microwave irradiation which presented higher reactivity, lower dipole moment, lower activation energy and stronger vibration around the carboxyl carbon, being more

Manuscript received December 4, 2014; revised March 15, 2015. This work was supported by the following Brazilian Goverment Agency: FAPEMIG (Grant \# CEX - APQ-04168-10 and PPM-00505-13).

Sabrina N. Rabelo, Leandro S. Oliveira, and Adriana S. Franca are with DEMEC, Universidade Federal de Minas Gerais, Av. Antônio Carlos 6627, Belo Horizonte, MG, 31270-901, Brazil (e-mail: sasanogueirarab@hotmail.com, leandro@demec.ufmg.br, adriana@demec.ufmg.br).

Vany P. Ferraz is with the Departamento de Química, Universidade Federal de Minas Gerais, Av. Antônio Carlos 6627, Belo Horizonte, MG, 31270-901, Brazil (e-mail: vanyferraz@ufmg.br). reactive than triolein with a higher dipole moment.

Microwave assisted transesterification of Pongamia pinnata seed oil was carried out by Kumar et al. [4] using methanol and the catalysts sodium hydroxide and potassium hydroxide. The experiments were carried out at 6:1 alcohol/oil molar ratio and $60^{\circ} \mathrm{C}$ reaction temperature. A significant reduction in reaction time for microwave induced transesterification was observed when compared to conventional heating. Tippayawong and Sittisun [5] studied a biodiesel production process from jatropha oil in a continuous flow with microwave heating, using sodium methoxide as a catalyst and with a microwave power of $800 \mathrm{~W}$. Irradiation time was varied between $10-40 \mathrm{~s}$ and the oil-to-methanol molar ratio was varied from 1:3 to $1: 9$ with increments of 3 regarding the number of moles of alcohol. $96.5 \%$ conversion of oil to biodiesel was obtained in $30 \mathrm{~s}$ using an oil-to-methanol molar ratio of 1:6 and 1\% catalyst. Lin et al. [6] studied the transesterification of palm oil in a microwave-irradiated reactor. The results indicated that the palm methyl ester yield first increased along with the amount of catalyst, reaction time, methanol-to-oil molar ratio, and reaction power, and then decreased as these parameters rose above certain levels. The best methyl ester yield was $99.5 \%$ with methyl ester content of $99.8 \%$ at 0.75 wt \% catalyst, a methanol-to-oil molar ratio of 6 , reaction time of $3 \mathrm{~min}$, and microwave power of $750 \mathrm{~W}$.

Other ongoing advances in the area of biodiesel are the developments of fast and reliable analytical methodologies for the determination of Fatty Acids Methyl Esters (FAME) concentrations in biodiesel. A plethora of analytical methodologies was developed for the analysis of biodiesel [7]-[11], with gas chromatography (GC) being the most commonly adopted technique as standard for the determination of FAME content in biodiesel by regulatory and monitoring agencies in the majority of countries [9]. However, chromatographic techniques, such as GC and high performance liquid chromatography, are time-consuming expensive techniques that demand qualified personnel and sample preparation for operation and the development of fast and reliable analytical techniques, that require little or no sample preparation at all for FAME quantification in biodiesel and its blends with fossil fuel, have become the major focus of several recent research works. Spectroscopic techniques such as Fourier Transform Infrared (FTIR) and near infrared (NIR) have gained special attention in recent years for they are fast analytical techniques that require no sample preparation for the analysis of biodiesel [11].

Partial least square regression (PLS) and artificial neural 
network (ANN) combined to attenuated total reflection Fourier Transform Infrared (ATR-FTIR) and NIR spectroscopies were used by Oliveira et al. [12] to design calibration models for the determination of methyl ester content in biodiesel blends with fossil diesel. Methyl esters were obtained by the methanolysis of soybean, babassu, dende, and soybean used frying oils. The PLS results have shown that the obtained models were suitable for the determination of diesel-biodiesel blends composed of methyl esters derived from different vegetable oils. Ferrão et al. [13] used PLS, interval partial least squares (iPLS) and synergy partial least squares (siPLS) regressions were used to simultaneous determination of quality parameters of biodiesel/diesel blends. Biodiesel amount, specific gravity, sulfur content and flash point were evaluated using spectroscopic data in the mid-infrared region obtained with an ATR accessory. The algorithms iPLS and siPLS were able to select the most adequate spectral region for each property studied. For all the properties studied, the siPLS algorithm produced better models than the full-spectrum PLS, selecting the most important bands. Mueller et al. [14] employed ATR-FTIR together with multivariate statistical analysis to identify vegetable oils used as raw material for biodiesel production. The vegetable oils canola, cotton, corn, palm, sunflower and soybean were used to produce biodiesel batches. For the multivariate analysis, principal component analysis (PCA), hierarchical cluster analysis (HCA), interval principal component analysis (iPCA) and soft independent modeling of class analogy (SIMCA) were used. The results indicated the feasibility of identifying vegetable oils used as raw material in the production of biodiesel by ATR-FTIR.

In view of the aforementioned, it was the aim of this work to develop a PLS model for the quantification of FAME in biodiesel produced by microwave-assisted transesterification of soybean oil with methanol and catalyzed by sodium methoxide.

\section{Methodology}

\section{A. Materials}

In this study, refined soybean oil was used as raw starting material and it was purchased from a local vendor (Belo Horizonte, Brazil). Its Free Fatty Acid (FFA) content was determined by a standard titration technique and its value was $0.6 \%(\mathrm{~m} / \mathrm{m})$. Laboratory grade methanol (100\%, Synth, Brazil) and sodium methoxide (30\% in methanol, ISOFAR, Brazil) were used as short chain alcohol and catalyst, respectively.

\section{B. Microwave Assisted Transesterification}

The transesterification of the oil was carried out in a glass reactor of $500 \mathrm{~mL}$ capacity in a microwave-irradiated unit (Model Start Synth, Milestone, Italy). The system was equipped with a reflux condenser, a magnetic stirrer bar and an infrared feedback temperature control system. For the transesterification under microwave heating, the catalyst concentration was varied from 1 to $3 \%$ (w/w of oil), the alcohol-to-oil molar ratio was varied from $3: 1$ to $12: 1$, by increments of 3 , and the reaction times studied were 1,7 and
15 minutes. The microwave unit was programmed to reach $60 \mathrm{oC}$ with a microwave power of $200 \mathrm{~W}$ (the maximum power output of the microwave unit is $1200 \mathrm{~W}$ ). The reaction was timed as soon as the system was turned on. All tests were conducted in duplicates.

\section{Separation and Purification of Biodiesel}

Upon reaching the desired time, the reaction was stopped by immediately immersing the glass reactor in an ice bath. Following the immersion in the ice bath, the mixture was transferred to a separating funnel and left to rest over night for the gravitational separation of the fatty acid methyl esters (FAME) and glycerol. After separation of the mixture in an upper layer of biodiesel and a lower glycerol layer, the upper layer (FAME) was collected for further purification. The adsorbent Amberlite BD10DRY was added to the FAME fraction (10\% weight in relation to the weight of the FAME) and put in an orbital shaker at $200 \mathrm{rpm}$ for 2 hours to remove the reminiscent water and free glycerin present in that phase. Afterwards, the purified FAME was subjected to a rotary evaporation to remove the methanol.

\section{Analytical Methods}

The purified FAME were analyzed by Attenuated Total Reflectance Fourier Transform Infrared (ATR-FTIR) and gas chromatography (GC). A Shimadzu IRAffinity-1 FTIR Spectrophotometer (Shimadzu, Japan) with a DLATGS (Deuterated Triglycine Sulfate Doped with L-Alanine) detector was used in the ATR-FTIR measurements that were performed in dry atmosphere $\left(18 \pm 0.5^{\circ} \mathrm{C}\right)$. A horizontal ATR sampling accessory (Shimadzu DRS8000A) equipped with $\mathrm{ZnSe}$ cell was employed. Approximately $22 \mathrm{mg}$ of the FAME samples were placed in the sampling accessory obtaining the best contact with the crystal. The empty accessory was used to obtain the background spectrum. The approximate total time required for spectral collection was $5 \mathrm{~min}$. All spectra were recorded within a range of 4000-600 $\mathrm{cm}^{-1}$ with a $4 \mathrm{~cm}^{-1}$ resolution and Happ-Genzel appodization. Each spectrum was calculated as the average of 20 scans and subjected to background subtraction. Analyses were carried out in triplicates and the average of the three were used to construct the models.

Gas chromatographic analyses were performed in accordance with EU norm EN 14103, using a GC-FID HP7820A apparatus (Agilent Technologies, USA) equipped with an autosampler (Agilent $7386 \mathrm{~B}$ series) and the data acquisition software EZChrom Elite Compact (Agilent Technologies, USA). Separations were accomplished using a 15-m long HP-INNOWAX capillary column, (0.25 mm I.D. and $0.25 \mu \mathrm{m}$ film thicknesses) at a constant hydrogen flow rate of $3 \mathrm{~mL} \mathrm{~min}^{-1}$. Samples $(1 \mu \mathrm{L})$ were injected in a split ratio of 1:50 with an injector temperature of $250^{\circ} \mathrm{C}$. The temperature program of the oven started with an initial temperature of $120^{\circ} \mathrm{C}$ and was followed by an increase in temperature up to $220^{\circ} \mathrm{C}$ at a rate of $7^{\circ} \mathrm{C} / \mathrm{min}$ for 10 minutes.

\section{E. Multivariate Statistical Analysis}

For the multivariate statistical analyses, a methyl oleate standard (Sigma-Aldrich, Brazil) was used in mixtures with the refined soybean oil in a way to cover the whole range of ester conversion, as presented in Table I. 
TABLE I: MiXTURES OF METHYL OLEATE STANDARD AND REFINED SOYBEAN OIL USED FOR THE MULTIVARIaTe STATISTICAL ANALYSES

\begin{tabular}{ccc}
\hline Sample & \% Methyl Oleate $(\mathbf{m} / \mathbf{m})$ & \% Soybean Oil $(\mathbf{m} / \mathbf{m})$ \\
\hline 1 & $0 \%$ & $100 \%$ \\
2 & $10 \%$ & $90 \%$ \\
3 & $20 \%$ & $80 \%$ \\
4 & $30 \%$ & $70 \%$ \\
5 & $40 \%$ & $60 \%$ \\
6 & $50 \%$ & $50 \%$ \\
7 & $60 \%$ & $40 \%$ \\
8 & $70 \%$ & $30 \%$ \\
9 & $80 \%$ & $20 \%$ \\
10 & $84 \%$ & $16 \%$ \\
11 & $88 \%$ & $12 \%$ \\
12 & $90 \%$ & $10 \%$ \\
13 & $92 \%$ & $8 \%$ \\
14 & $96 \%$ & $4 \%$ \\
15 & $100 \%$ & $0 \%$ \\
\hline
\end{tabular}

Principal Component Analysis (PCA) of all the obtained spectra for the mixtures of methyl oleate standard and refined soybean oil was spectra was performed to verify the capability of the multivariate analysis approach to discriminate the different samples with different contents of FAME. The 15 samples of mixtures were divided in 4 groups, taking as a reference the percentage of methyl oleate in each sample: 0 to $30 \%$; 31 to $60 \%$; 61 to $90 \%$; and 91 to $100 \%$. Partial least squares (PLS) regression analyses of the average ATR-FTIR spectra of the samples were carried out using MATLAB software, V. 7.13 (The MathWorks, Natick, Massachusetts, USA) and the PLS Toolbox (Eigenvector Technologies, Manson, USA). Cross-validation by a leave-one-out procedure was performed during the validation step to define the optimal number of parameters that should be kept in the model to detect outliers. The data were pre-processed by Standard Normal Variance (SNV) and Multiplicative Signal Correction (MSC) [15]. In addition, mean-centering and autoscaling methods were employed. Mean-centering method resulted in better regression models. The set of samples was divided into 2 groups with $30 \%$ being used for validation and $70 \%$ being used for calibration of the PLS regression model. The model incurring minimal error was selected, that is, the model with the lowest root mean square error of cross-validation (RMSECV) and lowest root mean square error of prediction (RMSEP).

\section{RESULTS AND DISCUSSION}

The ester concentrations for the microwave-assisted transesterification reactions are presented in Table II, as determined by $\mathrm{CG}$ analysis. It is clearly seen that the microwave-assisted transesterification of oils is a rather efficient process when compared to the conventional process, which is carried out in a batch stirred tank reactor and for which the reaction times required for the same magnitude of ester conversion are in the range of 60 to 120 minutes [1]. For the conditions of catalyst concentration of $3 \%$, reaction time of 1 minute and oil:alcohol molar ratio of $1: 6$, the ester concentration reached a value of $99.0 \pm 0.5 \%$, attesting the efficacy of the microwave-assisted process. Only two sets of conditions led to ester concentration values below the European and Brazilian specifications of ester concentration for commercialization of biodiesel that is $96.5 \%(\mathrm{~m} / \mathrm{m})$ : (1) Catalyst concentration of $1 \%$, reaction time of $7 \mathrm{~min}$ and oil:alcohol molar ratio of 1:6; and (2) catalyst concentration of $3 \%$, reaction time of $7 \mathrm{~min}$ and oil:alcohol molar ratio of $1: 12$. In the first case, the catalyst concentration of $1 \%$ has proven not to be enough for the desired conversion of oil into alkyl esters of fatty acids, even with the high intensity microwave energy used, and, in the second case, the excessive amount of methanol have proven to be a factor contributing to hinder the conversion for the reaction time employed.

TABLE II: ESTER CONCENTRATIONS FOR MICROWAVE-ASSISTED TRANSESTERIFICATION OF OILS AS DETERMINED BY CG ANALYSIS

\begin{tabular}{ccccc}
\hline Sample* & Molar Ratio (oil:alcohol) & \% Catalyst $\left(\mathbf{m} / \mathbf{m}_{\mathbf{o i l}}\right)$ & Reaction Time (min) & Ester Content $(\boldsymbol{\%} \mathbf{~ m} / \mathbf{m})$ \\
\hline 1C & $1: 6$ & 1 & 7 & $91.2 \pm 0.8$ \\
2C & $1: 6$ & 2 & 7 & $98.6 \pm 0.3$ \\
3C & $1: 6$ & 3 & 7 & $100.0 \pm 0.3$ \\
1T & $1: 6$ & 3 & 1 & $99.0 \pm 0.5$ \\
7T & $1: 6$ & 3 & 7 & $99.5 \pm 0.4$ \\
15T & $1: 6$ & 3 & 15 & $98.4 \pm 0.7$ \\
MR 1:3 & $1: 3$ & 3 & 7 & $99.0 \pm 0.3$ \\
MR 1:6 & $1: 6$ & 3 & 7 & $100.0 \pm 0.2$ \\
MR 1:9 & $1: 9$ & 3 & 7 & $98.4 \pm 0.6$ \\
MR 1:12 & $1: 12$ & 3 & 7 & $95.7 \pm 0.6$ \\
\hline
\end{tabular}

*C means catalyst concentration was varied and reaction time and oil:alcohol molar ratio remained constant at 7 min and 1:6, respectively; T means reaction time was varied and catalyst concentration and oil:alcohol molar ratio remained constant at 3\% and 1:6, respectively; and MR means oil:alcohol ratio was varied and catalyst concentration and reaction time remained constant at $3 \%$ and $7 \mathrm{~min}$, respectively.

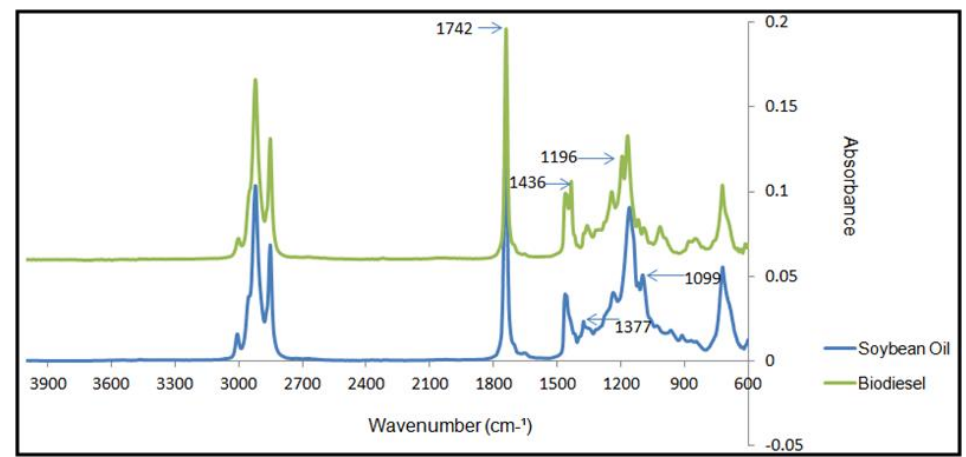

Fig. 1. ATR-FTIR spectra for a sample of refined soybean oil and of FAME of the same oil. 
The ATR-FTIR spectra for a sample of the refined soybean oil and for the FAME obtained with the conditions of catalyst concentration of $3 \%$, reaction time of 1 minute and oil:alcohol molar ratio of 1:6 are presented in Fig. 1. Rather subtle differences can be observed between the spectra, since the product of the transesterification process (FAME) is chemically similar to its precursor (the refined oil). In the region from $1800-1700 \mathrm{~cm}^{-1}$, it can be observed peaks that can be attributed to the stretching of $\mathrm{C}=\mathrm{O}$, typical of esters, and thus are common in both FAME and refined oil spectra [16]. The main spectrum region that allows for chemical discrimination between soybean oil and its respective FAME is in the range 1500-900 $\mathrm{cm}^{-1}$, known as "fingerprint" region. The peak at $1446 \mathrm{~cm}^{-1}$ correspond to the asymmetric stretching of $-\mathrm{CH}_{3}$ present in the biodiesel spectrum and absent in the refined oil spectrum (Soares et al., 2008). The peak at $1377 \mathrm{~cm}^{-1}$ can be attributed to the glycerol group $\mathrm{O}-\mathrm{CH}_{2}$ (mono-, di- and triglycerides), which is present in the refined oil spectrum and should be absent in the FAME spectrum [17]. The stretching of $\mathrm{O}-\mathrm{CH}_{3}$, represented by the absorbance at $1196 \mathrm{~cm}^{-1}$, is typical of biodiesel. Another region that allows for discrimination of FAME and refined oil is $1075-1100 \mathrm{~cm}^{-1}$, covering the asymmetric axial stretching of $\mathrm{O}-\mathrm{CH}_{2}-\mathrm{Ce}-\mathrm{CH}_{2}-\mathrm{OH}$, with respective peaks present only in the refined oil spectrum.

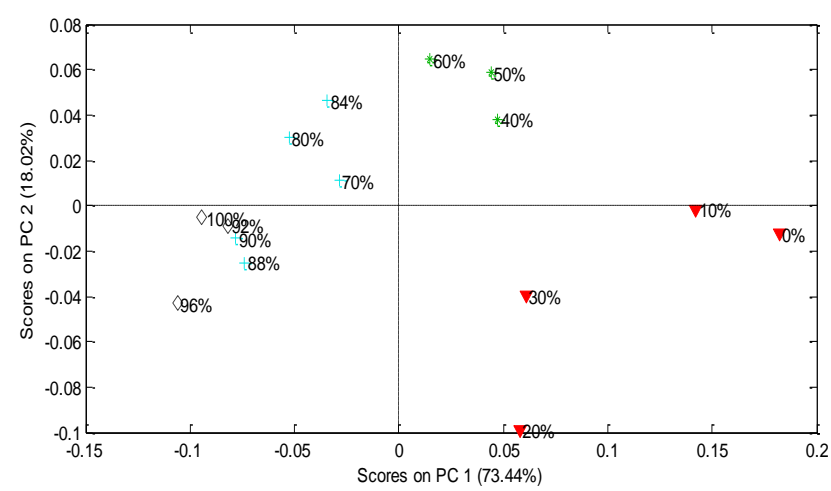

(a)

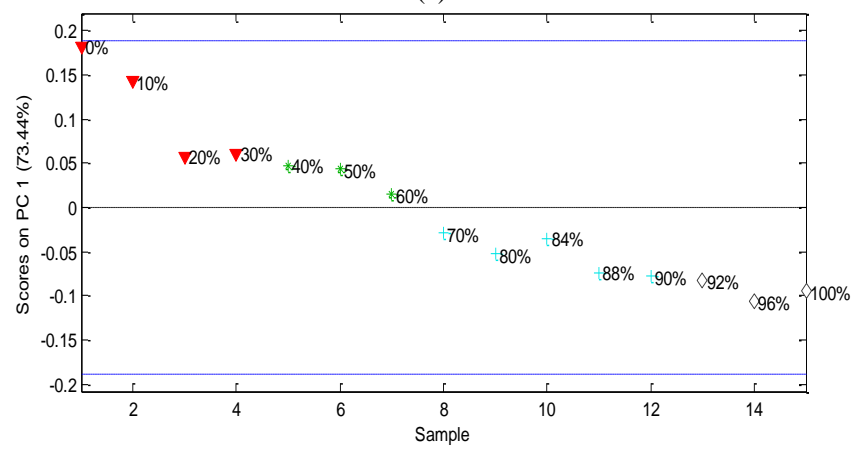

(b)

Fig. 2. Plots of (a) PC $1 \times$ PC 2 scores and (b) PC1 scores $\times$ samples: $\nabla 0$ to $30 \%, * 31$ to $60 \%,+61$ to $90 \%, \diamond 91$ to $100 \%$ FAME (represented by methyl oleate standard).

PCA of all the spectra for the mixtures of methyl oleate standard and refined soybean oil spectra was performed to verify the capability of the multivariate analysis approach to discriminate the different samples with different contents of FAME. The scatter plots obtained by PCA are displayed in Fig. 2(a). A clear separation between categories can be observed, with highest concentrations of FAME (ranging from 90 to $100 \%$ ) being clustered altogether in the negative quadrants of PC1 and PC2. PC1 and PC2 together explain $91.46 \%$ of the total variance amongst the samples. From the scores, it is observed there is a tendency of showing the evolution of the concentration as it increases from 0 to $100 \%$ (Fig. 2(a)). Fig. 2(b) presents the PC1 score plotted against the samples, clearly demonstrating the evolution of the FAME concentration as it increases from 0 to $100 \%$, as it happens during the transesterification reaction of the oil. Results from the principal components analysis indicate that the obtained spectra could provide enough information to develop quantification models for the different FAME concentrations.

Evaluation of the PC1 loadings plot (not shown) indicated that the spectral range that presented the highest influence on sample grouping was $1800-600 \mathrm{~cm}^{-1}$. Group separation was not improved by taking derivatives of the spectra.

Partial Least-Squares (PLS) regression was used to built FAME concentration quantification models for both the whole mid-infrared spectrum and the narrow region ranging from $1800-600 \mathrm{~cm}^{-1}$ that contributed most to the separation of distinct FAME concentration group in the PCA. Table III shows the results of RMSECV, RMSEP, number of latent variables, the Calibration Correlation Coefficient Rc, and the Validation Correlation Coefficient Rv for both the Standard Normal Variance (SNV) and Multiplicative Signal Correction (MSC) pre-treatments used in the full-spectrum PLS model for the quantification of FAME in biodiesel.

\begin{tabular}{|c|c|c|c|c|c|c|}
\hline $\begin{array}{l}\text { Type of Pre-Processing } \\
\text { (whole spectrum) }\end{array}$ & RMSEC (\%) & RMSECV (\%) & $\operatorname{RMSEP}(\%)$ & LV & Rc & $\mathrm{Rv}$ \\
\hline MC/SNV & 2.07484 & 4.31705 & 4.43876 & 3 & 0.996 & 0.969 \\
\hline MC/SNV & 0.57031 & 3.85306 & 3.75391 & 5 & 1.000 & 0.977 \\
\hline MC/MSC & 2.97777 & 4.57190 & 6.12287 & 2 & 0.992 & 0.992 \\
\hline MC/MSC & 1.01879 & 5.83188 & 4.42910 & 5 & 0.999 & 0.968 \\
\hline
\end{tabular}

MC: Mean-Centered; SNV: Standard Normal Variance; MSC Multiplicative Signal Correction; VL: Latent Variables; Rc: Calibration Correlation Coefficient; Rv: Validation Correlation Coefficient.

The best model was selected based on RMSEC and RMSEP. The lower their value the better the predictive capacity of the model. From Table III, it can be observed that the spectra subjected to the pre-treatment SNV, with mean-centered data, presented better results (i.e., lowest RMSEC and RMSEP) when compared to the spectra subjected to MSC for the same number of latent variables. The model based on the pre-treatment SNV with 5 latent variables (total of $99.91 \%$ of the spectral information) was the best model obtained for the quantification of FAME in biodiesel.

Based on the regression coefficients $\mathrm{Rc}$ and $\mathrm{Rv}$ of the model obtained for the pre-treatments SNV/Mean-Centered data and $5 \mathrm{LVs}$, the spectral region that presented the most influence on the discrimination of distinct FAME concentration groups was in the range of 1800-600 cm-1. Thus, this spectral region was used to build another PLS quantification model and the results for its parameters are presented in Table IV. In general, the models obtained for the 
spectral region of $1800-600 \mathrm{~cm}^{-1}$ were better than those obtained for the full spectrum. Again, the best results (i.e., lowest RMSEC and RMSEP) were obtained using the pre-treatments SNV/Mean-Centered data.

Fig. 3 shows a strong correlation between reference (determined by GC method) and predicted values for both calibration and validation sets, using the best PLS model obtained that was for the spectral region of $1800-600 \mathrm{~cm}^{-1}$ with the pre-treatments SNV and mean-centered data and 3 latent variables (Table IV).

TABLE IV: RESULTS FOR PLS MODEL FOR FAME QUANTIFICATION IN BIODIESEL USING MEAN-CENTERED DATA OF THE SPECTRAL REGION $1800-600 \mathrm{CM}^{-1}$

\begin{tabular}{ccccccc}
\hline $\begin{array}{c}\text { Type of Pre-Processing } \\
\left.\text { (spectral region of 1800-600 } \mathbf{c m}^{-1}\right)\end{array}$ & $\begin{array}{c}\text { RMSEC } \\
(\%)\end{array}$ & $\begin{array}{c}\text { RMSECV } \\
(\%)\end{array}$ & $\begin{array}{c}\text { RMSEP } \\
(\%)\end{array}$ & LV & Rc & Rv \\
\hline SNV/NC & 1.76970 & 3.72314 & 2.10461 & 3 & 0.997 & 0.993 \\
SNV/MC & 0.71299 & 3.90567 & 3.8013 & 5 & 1.000 & 0.983 \\
MSC/MC & 2.72740 & 3.94164 & 4.48099 & 2 & 0.993 & 0.998 \\
MSC/MC & 0.58449 & 4.71866 & 6.98227 & 5 & 1.000 & 0.965 \\
\hline
\end{tabular}

MC: Mean-Centered; SNV: Standard Normal Variance; MSC Multiplicative Signal Correction; VL: Latent Variables; Rc: Calibration Correlation Coefficient; Rv: Validation Correlation Coefficient.

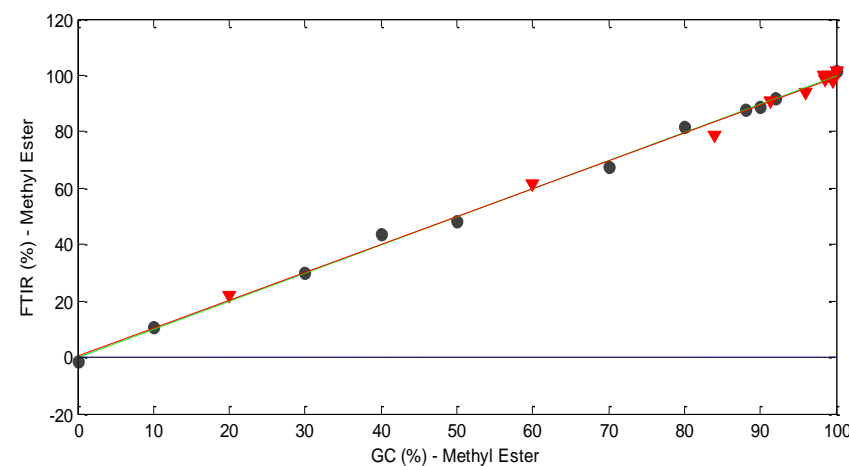

Fig. 3. FAME content $(\% \mathrm{~m} / \mathrm{m})$ vs. predicted values by cross-validation for the PLS model obtained for the spectral region of $1800-600 \mathrm{~cm}^{-1}$ with the pre-treatments SNV and mean-centered data and 3 latent variables: calibration samples and $\nabla$ validation samples.

Although some of the predicted values presented a little discrepancy regarding the reference values, the obtained model can be considered rather good for the quantification of FAME in biodiesel produced by microwave-assisted transesterification reactions. A more robust model can be built if non-converted mono and diglycerides are to be considered in the samples used for both calibration and validation. Nevertheless, considering that ATR-FTIR does not require sample preparation for the analysis, as opposed to the GC reference method adopt by most of the official methods from regulating and monitoring agencies in the world, it can be concluded that it is a rather promising candidate for becoming a reference analytical technique.

\section{REFERENCES}

[1] B. Bharathiraja, M. Chakravarthy, R. R. Kumar, D. Yuvaraj, J. Jayamuthunagai, R. P. Kumar, and S. Palani, "Biodiesel production using chemical and biological methods - A review of process, catalyst, acyl acceptor, source and process variables," Renewable and Sustainable Energy Reviews, vol. 38, pp. 368-382, October 2014.

[2] F. Motasemi and F. N. Ani, "A review on microwave-assisted production of biodiesel," Renewable and Sustainable Energy Reviews, vol. 16, pp. 4719-4733, June 2012.
[3] Y. Asakuma, Y. Ogawa, K. Maeda, K. Fukui, and H. Kuramochi, "Effects of microwave irradiation on triglyceride transesterification: Experimental and theoretical studies," Biochemical Engineering Journal, vol. 58-59, pp. 20-24, 2011.

[4] R. Kumar, G. R. Kumar, and N. Chandrashekar, "Microwave assisted alkali-catalyzed transesterification of Pongamia pinnata seed oil for biodiesel production," Bioresource Technology, vol. 102, pp 6617-6620, 2011.

[5] N. Tippayawong and P. Sittisun, "Continuous-flow transesterification of crude jatropha oil with microwave irradiation," Scientia Iranica, vol. B19, pp. 1324-1328, 2012.

[6] Y. C. Lin, K. H. Hsu, and J. F. Lin, "Rapid palm biodiesel production assisted by a microwave system and sodium methoxide catalyst," Fuel, vol. 115 , pp. 306-311, 2014.

[7] G. Knothe, "Analytical methods used in the production and fuel quality assessment of biodiesel," Transactions of the ASAE, vol. 44, pp. 193-200, 2001.

[8] M. R. Monteiro, A. R. P. Ambrozina, L. M. Lião, and A. G. Ferreira, "Critical review on analytical methods for biodiesel characterization," Talanta, vol. 77, pp. 593-605, 2008.

[9] M. Yahaya, I. Demshemino, I. Nwadike, S. O’Donnell, and L. Okoro "A review on the chromatographic analysis of biodiesel," International Journal of Education and Research, vol. 1, pp. 1-12, August 2013.

[10] W. Zhang, "Review on analysis of biodiesel with infrared spectroscopy," Renewable and Sustainable Energy Reviews, vol. 16, pp. 6048-6058, 2012.

[11] S. O’Donnell, I. Demshemino, M. Yahaya, I. Nwadike, and L. Okoro, "A review on the spectroscopic analyses of biodiesel," European International Journal of Science and Technology, vol. 2, pp. 137-146, September 2013

[12] J. S. Oliveira, R. Montalvão, L. Daher, P. A. Z. Suarez, and J. C. Rubim, "Determination of methyl ester contents in biodiesel blends by FTIR-ATR and FTNIR spectroscopies," Talanta, vol. 69, pp 1278-1284, 2006.

[13] M. F. Ferrão, M. S. Viera, R. E. P. Pazos, D. Fachini, A. E. Gerbase, and L. Marder, "Simultaneous determination of quality parameters of biodiesel/diesel blends using HATR-FTIR spectra and PLS, iPLS or siPLS regressions," Fuel, vol. 90, pp. 701-706, 2011.

[14] D. Mueller, M. F. Ferrão, L. Marder, A. B. Costa, and R. C. S. Schneider, "Fourier transform infrared spectroscopy (FTIR) and multivariate analysis for identification of different vegetable oils used in biodiesel production," Sensors, vol. 13, pp. 4258-4271, 2013.

[15] B. M. Wise, N. B. Gallagher, R. Bro, J. M. Shaver, W. Willem, and R. S. Koch, "PLS toolbox version 4.0 for use with Matlab ${ }^{\mathrm{TM}}$," Eigenvector Research, Inc., Wenatchee, USA, 2006.

[16] I. P. Soares, T. F. Rezende, R. C. Silva, E. V. R. Castro, and I. C. P Forte, "Multivariate calibration by variable selection for blends of raw soybean oil/biodiesel from different sources using Fourier Transform Infrared spectroscopy (FTIR) spectra data," Energy Fuels, vol. 22, pp. 2079-2083, January 2008.

[17] M. A. Dube, S. Zheng, D. D. Mclean, and M. J. A. Kates, "Comparison of attenuated total reflectance-FTIR spectroscopy and GPC for monitoring biodiesel production," Journal of the American Oil Chemist Society, vol. 81, pp. 599-603, June 2004.

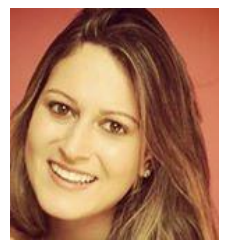

Sabrina N. Rabelo was born in 1987 and graduated in chemical engineering from Centro Universitário de Belo Horizonte (UNIBH), Belo Horizonte, Brazil, in 2012. She is currently enrolled in a Mechanical Engineering Graduate Program at Universidade Federal de Minas Gerais (UFMG) to obtain her master degree.

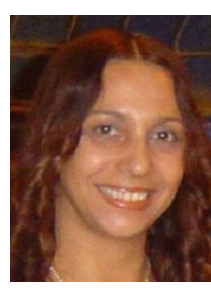

Vany P. Ferraz was born in 1962. She graduated in chemistry in 1987 and obtained her M.Sc. degree in chemistry in 1991 from Universidade Federal de Minas Gerais, Belo Horizonte, Brazil. She completed her Ph.D. degree in separation science from Ghent University, Belgium, in 1995.

She is currently working as an associate researcher at the Chemistry Department from Universidade Federal de Minas Gerais. She has published over 45 articles in international journals, 2 book chapters and has presented several research papers at various international conferences held in Brazil and abroad. Her research interests include gas chromatography, liquid chromatography, mass spectrometry and others. 


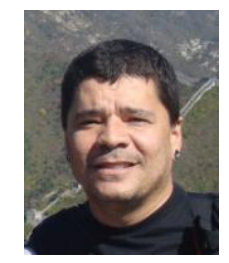

Leandro S. Oliveira was born in 1964. He graduated in chemical engineering in 1988 and obtained his M.Sc. degree in mechanical engineering in 1991 from Universidade Federal de Minas Gerais, Belo Horizonte, Brazil. He completed his Ph.D. degree in agricultural and biological engineering from Purdue University, USA, in 1995.

$\mathrm{He}$ is currently working as an associate professor at the Department of Mechanical Engineering from Universidade Federal de Minas Gerais and is currently the head of the Graduate Program at that Department. Dr. Oliveira also teaches at the graduate course in food sciences. He has published 65 articles in international journals, 11 book chapters and has presented several research papers at various international conferences held in Brazil and abroad. His research interests include sustainable uses of solid residues, biofuels, sustainable materials, coffee chemistry and others.

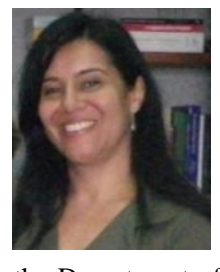

Adriana S. Franca was born in 1966. She graduated in chemical engineering in 1988 and obtained her M.Sc. degree in mechanical engineering in 1991 from Universidade Federal de Minas Gerais, Belo Horizonte, Brazil. She completed her Ph.D. degree in agricultural and biological engineering from Purdue University, USA, in 1995.

She is currently working as an associate professor at the Department of Mechanical Engineering from Universidade Federal de Minas Gerais and is a professor at the graduate course in food sciences. She has published over 75 articles in international journals, 16 book chapters and has presented several research papers at various international conferences held in Brazil and abroad. Her research interests include sustainable uses of agricultural residues, coffee chemistry, microwaves, FTIR and others. 